6. Internet resource: http: //journal.puet.edu.ua/index.php/nven/article/viewFile/ 349/369

7. Internet resource: http: //tourlib.net/books_green/gorishevsky6-3.htm

УДК 621.01

DOI: $10.31339 / 2617-0833-2018-2(25-30-38$

АНІМАЦІЯ КРИВОШИПНО - ПОВЗУННОГО МЕХАНІЗМУ ПРЕСА В ПАКЕТI МАТНСАD

Ігнатишин М. І., Кий I. O.

\title{
ANIMATION OF CRANK-SLIDING MECHANISM OF PRESS IN MATHCAD SYSTEM
}

Ihnatyshyn Mykola, Kyi Ivan

У даній роботі викладено побудову анімаційної моделі кривошипно-повзунного механізму в пакеті Mathcad 2001 Professional. 3 точки зору методики викладання теоріі механізмів і машин доцільно використати саме МаthCad, оскільки цей пакет передбачає трансформацію математичної моделі механізму у візуальну модель.

Ключові слова: кривошипно-повзунний механізм, анімація, mathcad.

The experience of teaching the theory of mechanisms and machines, in particular, shows that one of the main problems in solving the problems of mechanics is the lack of development of students' imagination. The process of describing the mechanical system involves predicting the directions of possible movements of the system and, on this basis, further choosing a method for its description.

Imagination in the simplest sense of the word is the process of drawing images from memory. In a productive sense, imagination refers to the process of re-structuring memory images from past experience and pre-formed images into new designs. That is, imagination is seen as creative and constructive thinking.

The animation of complex mechanical systems with repetitive motions in itself represents an interest in terms of animation of the imagination, its training, as a means of solving the contradiction between the expected movement and the motion of the system on the screen. Interactive elements that allow changing the parameters of the system and, thus, the nature of the movement, serve as an additional catalyst for the work of the imagination.

An essential place in the teaching of fundamental and technical disciplines is visualization of educational material.

Modern computer software systems provide great opportunities for visualizing educational material and intensifying the teaching process in classes.

Libraries Compass, Autocad, SolidWorks allow simulating the movements of parts of the product in the process of real work.

The use of computer packages: MatLab, Maple, Mathematica, MathCad leads to the need for fundamental changes in the method of conducting practical classes and the content of the lecture material.

The mathematical model of the crank-sliding mechanism consists of equations that describe the position of the points and parts of the investigated lever mechanism from the angle determining the position of the input link - the crank.

In this paper, the construction of an animation model of the crank-sliding mechanism in the package Mathcad 2001 Professional has been presented. From the point of view of the technique of teaching the theory of mechanisms and machines it is expedient to use MathCad, since this package involves the transformation of the mathematical model of the mechanism into a visual model. 


\section{Keywords: crank-sliding mechanism, animation, mathcad.}

Комп'ютерне моделювання застосовується в різних галузях: математика, фізика, біологія, екологія, астрономія та інші, і воно є одним із найбільш успішних методів дослідження складних систем.

У наш час одержали широке застосування такі потужні пакети математичного моделювання, які не вимагають спеціальних знань в програмуванні, це Maple, MatLab, Mathcad, SolidWorks тощо [1,2]. Полегшуючи розв'язання складних математичних задач, такі системи дозволяють зняти психологічний бар'єр при вивчені механіки, математики, опору матеріалів, теорії механізмів і машин, а також роблять цей процес більш цікавим i простим, значно підвищують ефективність праці різних спеціалістів. Грамотне їх використання у навчальному процесі дозволяє підвищити рівень математичної та технічної освіти $[3,5]$.

Роль та важливість сучасних комп'ютерно-інформаційних технологій сьогодні важко переоцінити. Особливої значимості набуває наявність фахівців в даній галузі на сучасному ринку праці. Потреба в даних спеціалістах зростає неймовірними кроками, їх нестача відчувається вже сьогодні. Тому, у спеціалізованих та не спеціалізованих навчальних закладах (професійні школи та вищі навчальні заклади) приділяють значну увагу вивченню сучасних інженерних систем, які базуються на комп’ютерному моделюванні, зокрема трансформації математичних формул, що описують об’єкт в анімацію.

Метою даної роботи $є$ анімація важільного механізму механічного пресу шляхом застосування векторної алгебри та пакету Mathcad 2001 Professional.

Об'єктом дослідження є кривошипно-повзунний механізм пресу, що складається 3 одної нерухомої ланки, - стояка, і п’ять рухомих ланок: кривошипа, 2-х шатунів, коромисла i повзуна. Кривошип здійснює обертальний рух, шатун приймає участь у двох рухах, поступальному та обертальному зі змінною кутовою швидкістю, коромисло здійснює коливні рухи, повзун рухається поступально.

Для дослідження кінематики механізму застосовано метод комп’ютерного моделювання та анімації в пакеті Mathcad 2001 Professional. для анімації.

Для кривошипно-повзунного механізму, рис.1., скласти програму, в системі Mathcad,

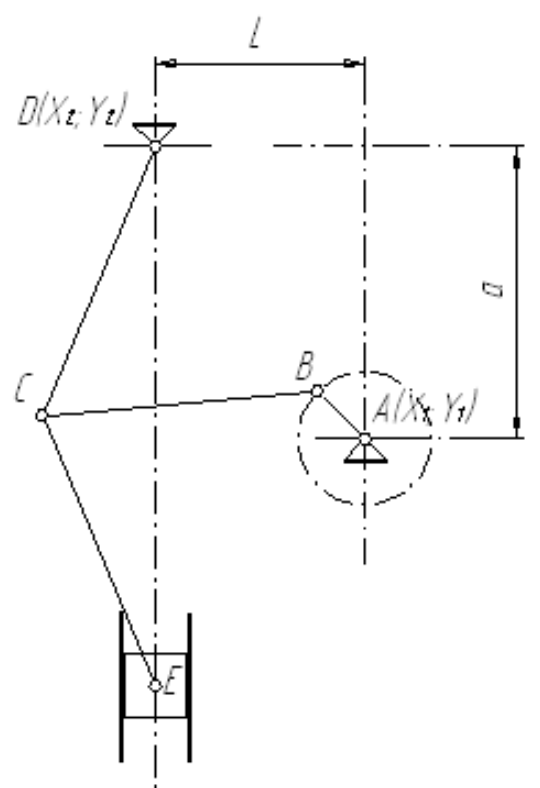

Рис. 1. Важільний механізм преса (АВ - кривошип, $\mathrm{BC}$ - шатун, $\mathrm{CD}$ - коромисло, $\mathrm{CE}$ - шатун, E - повзун). 


\section{Результати математичного моделювання.}

Початок програми «Анімація важільного механізму преса».

Для аналітичного дослідження важільного механізму поршневого насоса застосуємо Mathcad. Mathcad-система комп'ютерної алгебри з класу систем проектування, орієнтована на підготовку інтерактивних документів з обчисленнями і візуальним супроводом.

Побудуємо програму на мові Mathcad.

Ввдемо декотрі оператори мови Mathcad X := ORIGIN, Y := ORIGIN + 1

Визначимо тригонометричні функції, кут в градусній мірі.

$$
\begin{gathered}
\arccos (\mathrm{x}):=\operatorname{acos}(\mathrm{x}) \cdot \mathrm{deg}^{-1}, \arcsin (\mathrm{x}):=\operatorname{asin}(\mathrm{x}) \cdot \mathrm{deg}^{-1}, \sin (\mathrm{x}):=\sin (\mathrm{x} \cdot \mathrm{deg}) \\
\cos (\mathrm{x}):=\cos (\mathrm{x} \cdot \operatorname{deg})
\end{gathered}
$$

Одиничні орти осей координат на площині: $\mathrm{e}_{\mathrm{X}}:=\left(\begin{array}{l}1 \\ 0\end{array}\right) \quad \mathrm{e}_{\mathrm{Y}}:=\left(\begin{array}{l}0 \\ 1\end{array}\right)$

Вектор (функція поворота вектора), що отримується поворотом вихідного вектора в площині без або зі зміною його довжини

r - вихідний вектор, який повертають;

a - кут повороту (+ якщо проти годинникової стрілки; - якщо за годинниковою стрілкою;

NovaLong - нова довжина вектора.

$$
\rho(\mathrm{r}, \alpha, \text { NovaLong }):=\left(\begin{array}{cc}
\cos (\alpha) & -\sin (\alpha) \\
\sin (\alpha) & \cos (\alpha)
\end{array}\right) \cdot\left(\begin{array}{c}
r_{X} \\
r_{Y}
\end{array}\right) \cdot \frac{\text { NovaLong }}{|r|} .
$$

Визначення кута між векторами L1 i L2 (за теоремою косинусів)

$$
\operatorname{Tcos}\left(\mathrm{L}_{1}, \mathrm{~L}_{2}, \mathrm{~L}_{3}\right):=\arccos \left(\frac{\mathrm{L}_{1}^{2}+\mathrm{L}_{2}^{2}-\mathrm{L}_{3}^{2}}{2 \cdot \mathrm{L}_{1} \cdot \mathrm{L}_{2}}\right) \text {. }
$$

Визначення орта $\operatorname{Ort}(\mathrm{r}):=\frac{\mathrm{r}}{|\mathrm{r}|}$.

Визначення аналога кутової швидкості вектора-ланки

$$
\omega_{\mathrm{a}}\left(\mathrm{r}, \mathrm{V}_{\mathrm{a}}\right):=\frac{\mathrm{r}_{\mathrm{X}} \cdot \mathrm{V}_{\mathrm{a}_{\mathrm{Y}}}-\mathrm{r}_{\mathrm{Y}} \cdot \mathrm{V}_{\mathrm{a}_{\mathrm{X}}}}{(|\mathrm{r}|)^{2}} .
$$

Визначення аналога кутового прискорення вектора-ланки

$$
\varepsilon_{a}\left(r, a_{a}\right):=\frac{r_{X} \cdot a_{a_{Y}}-r_{Y} \cdot a_{a_{X}}}{(|r|)^{2}} .
$$

Функція відрізка-лінії на площині $\operatorname{Line}\left(\mathrm{V}_{1}, \mathrm{~V}_{2}, \mathrm{~K}\right):=\left(\begin{array}{c}\mathrm{V}_{1_{\mathrm{K}}} \\ \mathrm{V}_{2_{\mathrm{K}}}\end{array}\right)$.

Вихідні дані, рис.1:

$\mathrm{a}:=700 \cdot \mathrm{mm}, \quad \mathrm{L}:=500 \cdot \mathrm{mm}, \quad \mathrm{AB}:=160 \cdot \mathrm{mm}, \quad \mathrm{BC}:=\mathrm{L}-\mathrm{AB} \quad(550 \cdot \mathrm{mm})$,

$\mathrm{CD}:=700 \cdot \mathrm{mm}, \mathrm{CE}:=700 \cdot \mathrm{mm}, \mathrm{D}_{\mathrm{n}}:=150 \cdot \mathrm{mm}, \mathrm{n}_{1}:=6$ об/хв

Координати точки $\mathrm{O}_{1}$ (стояк) $\mathrm{X}_{1}:=0 \cdot \mathrm{mm}, \mathrm{Y}_{1}:=0 \cdot \mathrm{mm}$. 
Координати точки $\mathrm{O}_{4}$ (стояк) $\mathrm{X}_{4}:=-\mathrm{L}, \mathrm{Y}_{4}:=\mathrm{a}$.

Довжина ланки 1-2 (кривошипа) $\mathrm{L}_{12}:=\mathrm{AB}$.

Довжина ланки 2-3 (шатун) $\quad \mathrm{L}_{23}:=\mathrm{BC}$.

Довжина ланки 3-4 (кривошипа) $\mathrm{L}_{34}:=\mathrm{CD}$.

Довжина ланки 3-5 (шатун) $\quad \mathrm{L}_{35}:=\mathrm{CE}$.

Варіант складання двоповодкової групи першого виду 2-3-4 $\mathrm{W}_{1}:=-1$

Початковий кут ланки 1-2 (кривошипа), град $\alpha_{0}:=0$

Кутова швидкість ланки 1-2 (кривошипа) $\Omega_{1}:=\frac{\mathrm{n}_{1} \cdot \pi}{30} \mathrm{~s}^{-1} \cdot \mathrm{deg}^{-1}$

Кутове прискорення ланки 1-2 (кривошипа)

Кількість положень механізму

$$
\mathrm{E}_{1}:=0 \mathrm{~s}^{-2} \cdot \mathrm{deg}^{-1}
$$

Максимальний кут повороту кривошипа

$$
\begin{array}{r}
\mathrm{N}_{\text {POL }}:=12 \\
\alpha_{\text {max }}:=\alpha_{0}+360
\end{array}
$$

Крок розрахунку

$$
\Delta \alpha:=\frac{\alpha_{\text {max }}-\alpha_{0}}{\mathrm{~N}_{\mathrm{POL}}}
$$

Поточний кут повороту ланки $1-2$ (кривошипа) $\alpha_{1}:=\alpha_{0}$ (або $\left.\alpha_{0}, \alpha_{0}+\Delta \alpha_{. .} \alpha_{\text {max }}\right)$

Центри мас ланок $\quad \mathrm{S}_{12}:=0.5 \cdot \mathrm{L}_{12} \quad, \quad \mathrm{~S}_{23}:=0.5 \cdot \mathrm{L}_{23}, \quad \mathrm{~S}_{34}:=0.5 \cdot \mathrm{L}_{34}$, $\mathrm{S}_{35}:=0.5 \cdot \mathrm{L}_{35}$

Маси та моменти інерції ланок

Ланка $\mathrm{AB}, \mathrm{m}_{1}:=0.134 \mathrm{~kg}, \mathrm{~J}_{1}:=2.15 \times 10^{-4} \mathrm{kgm}^{2}$, ланка $\mathrm{BC}, \mathrm{m}_{2}:=\mathrm{m}_{2}$, $\mathrm{J}_{2}:=0.015 \mathrm{kgm}^{2}$, ланка $\mathrm{CD}, \mathrm{m}_{3}:=0.588 \mathrm{~kg}, \mathrm{~J}_{3}:=0.018 \mathrm{kgm}^{2}$, ланка CE, $\mathrm{m}_{4}:=0.588 \mathrm{~kg}, \mathrm{~J}_{4}:=0.018 \mathrm{kgm}^{2}$, ланка $\mathrm{E}, \mathrm{m}_{5}:=0.126 \mathrm{~kg}$.

\section{Розрахунок.}

Вирази для визначення векторів-точок, векторів-ланок та кутів між ланками.

Вектори-точки стояків $\mathrm{P}_{\mathrm{O} 1}:=\left(\begin{array}{c}\mathrm{X}_{1} \\ \mathrm{Y}_{1}\end{array}\right) \mathrm{P}_{\mathrm{O} 4}:=\left(\begin{array}{c}\mathrm{X}_{4} \\ \mathrm{Y}_{4}\end{array}\right)$.

Вектори-функції кута повороту вхідної ланки, кривошипа $\mathrm{O}_{1} \mathrm{~A}, \alpha_{1}$ :

Вектор-ланка та вектор-точка шарніра

$$
\mathrm{P}_{12}\left(\alpha_{1}\right):=\rho\left(\mathrm{e}_{X}, \alpha_{1}, \mathrm{~L}_{12}\right), \mathrm{P}_{2}\left(\alpha_{1}\right):=\mathrm{P}_{\mathrm{O} 1}+\mathrm{P}_{12}\left(\alpha_{1}\right)
$$

Вектор-змінної довжини

$$
\mathrm{P}_{24}\left(\alpha_{1}\right):=\mathrm{P}_{\mathrm{O} 4}-\mathrm{P}_{2}\left(\alpha_{1}\right)
$$

Кут $\phi_{243}$ між вектором змінної довжини $\mathrm{P}_{24}$ та вектором-ланкою коромислом 
$\mathrm{P}_{34}$ ( з модулем $\mathrm{L}_{34}$ ).

$$
\phi_{243}\left(\alpha_{1}\right):=\operatorname{T} \cos \left(\left|\mathrm{P}_{24}\left(\alpha_{1}\right)\right|, \mathrm{L}_{34}, \mathrm{~L}_{23}\right)
$$

Вектори-ланки коромисло $\mathrm{P}_{34}, \mathrm{P}_{23}, \mathrm{P}_{35}$ та вектори-точки $\mathrm{P}_{3}, \mathrm{P}_{5}$

$$
\begin{gathered}
\mathrm{P}_{34}\left(\alpha_{1}\right):=\rho\left(\mathrm{P}_{24}\left(\alpha_{1}\right), \mathrm{W}_{1} \cdot \phi_{243}\left(\alpha_{1}\right), \mathrm{L}_{34}\right), \mathrm{P}_{23}\left(\alpha_{1}\right):=\mathrm{P}_{24}\left(\alpha_{1}\right)-\mathrm{P}_{34}\left(\alpha_{1}\right) \\
\mathrm{P}_{3}\left(\alpha_{1}\right):=\mathrm{P}_{2}\left(\alpha_{1}\right)+\mathrm{P}_{23}\left(\alpha_{1}\right), \\
\mathrm{P}_{5}\left(\alpha_{1}\right):=-\mathrm{L} \cdot \mathrm{e}_{\mathrm{X}}-\left[\left|\mathrm{P}_{34}\left(\alpha_{1}\right) \mathrm{Y}\right|-\mathrm{P}_{\mathrm{O} 4}+\sqrt{\mathrm{L}_{35}{ }^{2}-\left(\left|\mathrm{P}_{34}\left(\alpha_{1}\right) \mathrm{X}\right|\right)^{2}}\right] \cdot \mathrm{e}_{\mathrm{Y}}, \\
\mathrm{P}_{35}\left(\alpha_{1}\right):=\mathrm{P}_{5}\left(\alpha_{1}\right)-\mathrm{P}_{3}\left(\alpha_{1}\right) .
\end{gathered}
$$

Вектори-точок центрів мас

$$
\begin{aligned}
\mathrm{S}_{12}\left(\alpha_{1}\right):=\mathrm{S}_{12} \cdot \frac{\mathrm{P}_{12}\left(\alpha_{1}\right)}{\left|\mathrm{P}_{12}\left(\alpha_{1}\right)\right|}, \mathrm{S}_{1}\left(\alpha_{1}\right):=\mathrm{P}_{\mathrm{O} 1}+\mathrm{S}_{12}\left(\alpha_{1}\right), \\
\mathrm{S}_{23}\left(\alpha_{1}\right):=\mathrm{S}_{23} \cdot \frac{\mathrm{P}_{23}\left(\alpha_{1}\right)}{\left|\mathrm{P}_{23}\left(\alpha_{1}\right)\right|}, \mathrm{S}_{2}\left(\alpha_{1}\right):=\mathrm{P}_{2}\left(\alpha_{1}\right)+\mathrm{S}_{23}\left(\alpha_{1}\right), \\
\mathrm{S}_{34}\left(\alpha_{1}\right):=\mathrm{S}_{34} \cdot \frac{\mathrm{P}_{34}\left(\alpha_{1}\right)}{\left|\mathrm{P}_{34}\left(\alpha_{1}\right)\right|}, \mathrm{S}_{3}\left(\alpha_{1}\right):=\mathrm{P}_{3}\left(\alpha_{1}\right)+\mathrm{S}_{34}\left(\alpha_{1}\right), \\
\mathrm{S}_{35}\left(\alpha_{1}\right):=\mathrm{S}_{35} \cdot \frac{\mathrm{P}_{35}\left(\alpha_{1}\right)}{\left|\mathrm{P}_{35}\left(\alpha_{1}\right)\right|}, \mathrm{S}_{4}\left(\alpha_{1}\right):=\mathrm{P}_{3}\left(\alpha_{1}\right)+\mathrm{S}_{35}\left(\alpha_{1}\right) .
\end{aligned}
$$

Анімація важільного механізму пресса.

Функції лінії-відрізки кута $\alpha_{1}$ :

a) Важільний механізм.

$$
\begin{gathered}
\mathrm{L}_{1}\left(\alpha_{1}, \mathrm{~K}\right):=\operatorname{Line}\left(\mathrm{P}_{\mathrm{O} 1}, \mathrm{P}_{2}\left(\alpha_{1}\right), \mathrm{K}\right), \\
\mathrm{L}_{2}\left(\alpha_{1}, \mathrm{~K}\right):=\operatorname{Line}\left(\mathrm{P}_{2}\left(\alpha_{1}\right), \mathrm{P}_{3}\left(\alpha_{1}\right), \mathrm{K}\right), \\
\mathrm{L}_{3}\left(\alpha_{1}, \mathrm{~K}\right):=\operatorname{Line}\left(\mathrm{P}_{3}\left(\alpha_{1}\right), \mathrm{P}_{\mathrm{O} 4}, \mathrm{~K}\right), \mathrm{L}_{4}\left(\alpha_{1}, \mathrm{~K}\right):=\operatorname{Line}\left(\mathrm{P}_{3}\left(\alpha_{1}\right), \mathrm{P}_{5}\left(\alpha_{1}\right), \mathrm{K}\right) \\
\mathrm{L}\left(\alpha_{1}, \mathrm{~K}\right):=\operatorname{augment}\left(\mathrm{L}_{1}\left(\alpha_{1}, \mathrm{~K}\right), \mathrm{L}_{2}\left(\alpha_{1}, \mathrm{~K}\right), \mathrm{L}_{3}\left(\alpha_{1}, \mathrm{~K}\right), \mathrm{L}_{4}\left(\alpha_{1}, \mathrm{~K}\right)\right) .
\end{gathered}
$$

б) Пресувальний повзун

$$
\begin{array}{r}
\mathrm{P}_{\mathrm{D} 1}\left(\alpha_{1}\right):=\mathrm{P}_{5}\left(\alpha_{1}\right)+\rho\left(\mathrm{e}_{\mathrm{X}}, 45, \frac{\sqrt{2}}{2} \cdot \mathrm{D}_{\mathrm{n}}\right), \\
\mathrm{P}_{\mathrm{D} 2}\left(\alpha_{1}\right):=\mathrm{P}_{5}\left(\alpha_{1}\right)+\rho\left(\mathrm{e}_{\mathrm{X}}, 135, \frac{\sqrt{2}}{2} \cdot \mathrm{D}_{\mathrm{n}}\right) \\
\mathrm{P}_{\mathrm{D} 3}\left(\alpha_{1}\right):=\mathrm{P}_{5}\left(\alpha_{1}\right)+\rho\left(\mathrm{e}_{\mathrm{X}}, 225, \frac{\sqrt{2}}{2} \cdot \mathrm{D}_{\mathrm{n}}\right),
\end{array}
$$




$$
\begin{gathered}
\mathrm{P}_{\mathrm{D} 4}\left(\alpha_{1}\right):=\mathrm{P}_{5}\left(\alpha_{1}\right)+\rho\left(\mathrm{e}_{\mathrm{X}}, 315, \frac{\sqrt{2}}{2} \cdot \mathrm{D}_{\mathrm{n}}\right) \\
\mathrm{L}_{6}\left(\alpha_{1}, \mathrm{~K}\right):=\operatorname{Line}\left(\mathrm{P}_{\mathrm{D} 1}\left(\alpha_{1}\right), \mathrm{P}_{\mathrm{D} 2}\left(\alpha_{1}\right), \mathrm{K}\right) \\
\mathrm{L}_{7}\left(\alpha_{1}, \mathrm{~K}\right):=\operatorname{Line}\left(\mathrm{P}_{\mathrm{D} 2}\left(\alpha_{1}\right), \mathrm{P}_{\mathrm{D} 3}\left(\alpha_{1}\right), \mathrm{K}\right), \\
\mathrm{L}_{8}\left(\alpha_{1}, \mathrm{~K}\right):=\operatorname{Line}\left(\mathrm{P}_{\mathrm{D} 3}\left(\alpha_{1}\right), \mathrm{P}_{\mathrm{D} 4}\left(\alpha_{1}\right), \mathrm{K}\right), \\
\mathrm{L}_{9}\left(\alpha_{1}, \mathrm{~K}\right):=\operatorname{Line}\left(\mathrm{P}_{\mathrm{D} 4}\left(\alpha_{1}\right), \mathrm{P}_{\mathrm{D} 1}\left(\alpha_{1}\right), \mathrm{K}\right), \\
\mathrm{L}_{\text {piston }}\left(\alpha_{1}, \mathrm{~K}\right):=\operatorname{augment}\left(\mathrm{L}_{6}\left(\alpha_{1}, \mathrm{~K}\right), \mathrm{L}_{7}\left(\alpha_{1}, \mathrm{~K}\right), \mathrm{L}_{8}\left(\alpha_{1}, \mathrm{~K}\right), \mathrm{L}_{9}\left(\alpha_{1}, \mathrm{~K}\right)\right) .
\end{gathered}
$$

в) Циліндр преса

Розрахунок ходу пресувального повзуна, $\mathrm{H}$.

3 графіка, рис.2, знаходимо наближені значення кутів $\alpha_{\min }$ та $\alpha_{\max }$ кривошипа $\mathrm{O}_{1}$ А, що відповідають крайнім положенням пресувального повзуна

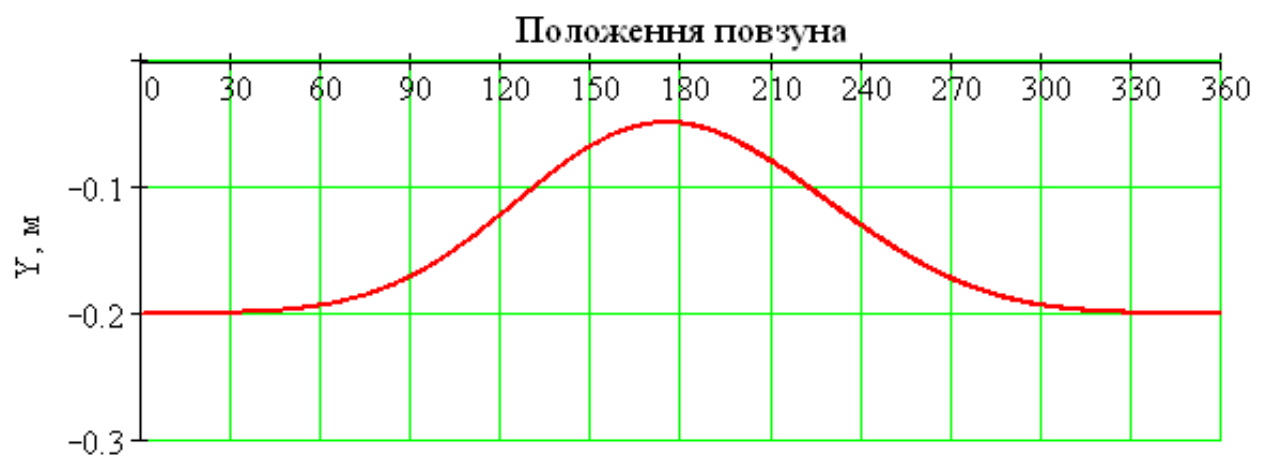

Кут повороту кривошипа в гадусах

Рис. 2. Графік залежності положення пресувального повзуна від кута $\alpha_{1}$.

Знаходимо точні значення кутів $\alpha_{\min }$ та $\alpha_{\max }$ кривошипа $\mathrm{O}_{1} \mathrm{~A}$, що відповідають крайнім положенням пресувального повзуна та хід пресувального повзуна Н.

$$
\begin{array}{r}
\mathrm{f}(\phi):=\frac{\mathrm{d}}{\mathrm{d} \phi} \mathrm{P}_{5}(\phi)_{\mathrm{Y}}, \phi_{1}:=10, \alpha_{\min }:=\operatorname{root}\left(\mathrm{f}\left(\phi_{1}\right), \phi_{1}\right), \alpha_{\min }=6.669 . \\
\mathrm{f}(\phi):=\frac{\mathrm{d}}{\mathrm{d} \phi} \mathrm{P}_{5}(\phi)_{\mathrm{Y}}, \phi_{2}:=190, \alpha_{\max }:=\operatorname{root}\left(\mathrm{f}\left(\phi_{2}\right), \phi_{2}\right), \alpha_{\max }=172.651 . \\
\mathrm{H}:=\mathrm{P}_{5}\left(\alpha_{\max }\right)_{\mathrm{Y}}-\mathrm{P}_{5}\left(\alpha_{\min }\right)_{\mathrm{Y}}, \mathrm{H}=0.151 \mathrm{~m}-\text { хід пресувального повзуна. }
\end{array}
$$

Координати центра циліндра

$$
\begin{aligned}
& \mathrm{X}_{0}:=\mathrm{P}_{5}\left(\alpha_{\max }\right) \mathrm{X}, \mathrm{X}_{0}=-0.5 \mathrm{~m} . \\
& \mathrm{Y}_{0}:=\frac{\mathrm{P}_{5}\left(\alpha_{\max }\right) \mathrm{Y}+\mathrm{P}_{5}\left(\alpha_{\min }\right) \mathrm{Y}}{2}, \mathrm{Y}_{0}=-0.624 \mathrm{~m} .
\end{aligned}
$$

Вектори-точки циліндра. Відстань між пресувальним повзуном та циліндром на схемі приймемо $\tau:=\frac{\mathrm{D}_{\mathrm{n}}}{10}$. 


$$
\begin{aligned}
& \mathrm{P}_{\mathrm{C} 1}\left(\alpha_{1}\right):=\left(\begin{array}{c}
\mathrm{X}_{0} \\
\mathrm{Y}_{0}
\end{array}\right)+\left(\begin{array}{c}
\frac{\mathrm{D}_{\mathrm{n}}}{2}+\tau \\
\frac{\mathrm{H}}{2}+\frac{\mathrm{D}_{\mathrm{n}}}{2}
\end{array}\right), \mathrm{P}_{\mathrm{C} 2}\left(\alpha_{1}\right):=\left(\begin{array}{c}
\mathrm{X}_{0} \\
\mathrm{Y}_{0}
\end{array}\right)+\left[\begin{array}{c}
-\left(\frac{\mathrm{D}_{\mathrm{n}}}{2}+\tau\right) \\
\frac{\mathrm{H}}{2}+\frac{\mathrm{D}_{\mathrm{n}}}{2}
\end{array}\right], \\
& \mathrm{P}_{\mathrm{C} 3}\left(\alpha_{1}\right):=\left(\begin{array}{c}
\mathrm{X}_{0} \\
\mathrm{Y}_{0}
\end{array}\right)+\left[\begin{array}{c}
-\left(\frac{\mathrm{D}_{\mathrm{n}}}{2}+\tau\right) \\
-\frac{\mathrm{H}}{2}-\frac{\mathrm{D}_{\mathrm{n}}}{2}
\end{array}\right], \mathrm{P}_{\mathrm{C} 4}\left(\alpha_{1}\right):=\left(\begin{array}{c}
\mathrm{X}_{0} \\
\mathrm{Y}_{0}
\end{array}\right)+\left(\begin{array}{c}
\frac{\mathrm{D}_{\mathrm{n}}}{2}+\tau \\
-\frac{\mathrm{H}}{2}-\frac{\mathrm{D}_{\mathrm{n}}}{2}
\end{array}\right) .
\end{aligned}
$$

Лінії-відрізки

$$
\begin{aligned}
& \mathrm{L}_{10}\left(\alpha_{1}, \mathrm{~K}\right):=\operatorname{Line}\left(\mathrm{P}_{\mathrm{C} 1}\left(\alpha_{1}\right), \mathrm{P}_{\mathrm{C} 4}\left(\alpha_{1}\right), \mathrm{K}\right) \\
& \mathrm{L}_{11}\left(\alpha_{1}, \mathrm{~K}\right):=\operatorname{Line}\left(\mathrm{P}_{\mathrm{C} 2}\left(\alpha_{1}\right), \mathrm{P}_{\mathrm{C} 3}\left(\alpha_{1}\right), \mathrm{K}\right) \\
& \mathrm{L}_{\text {cilinder }}\left(\alpha_{1}, \mathrm{~K}\right):=\operatorname{augment}\left(\mathrm{L}_{10}\left(\alpha_{1}, \mathrm{~K}\right), \mathrm{L}_{11}\left(\alpha_{1}, \mathrm{~K}\right)\right)
\end{aligned}
$$

Визначення допоміжної ранжируваної змінної $\boldsymbol{F}$ для візуалізації та анімації кінематичної схеми механізму $\mathrm{F}:=\mathrm{FRAME}$

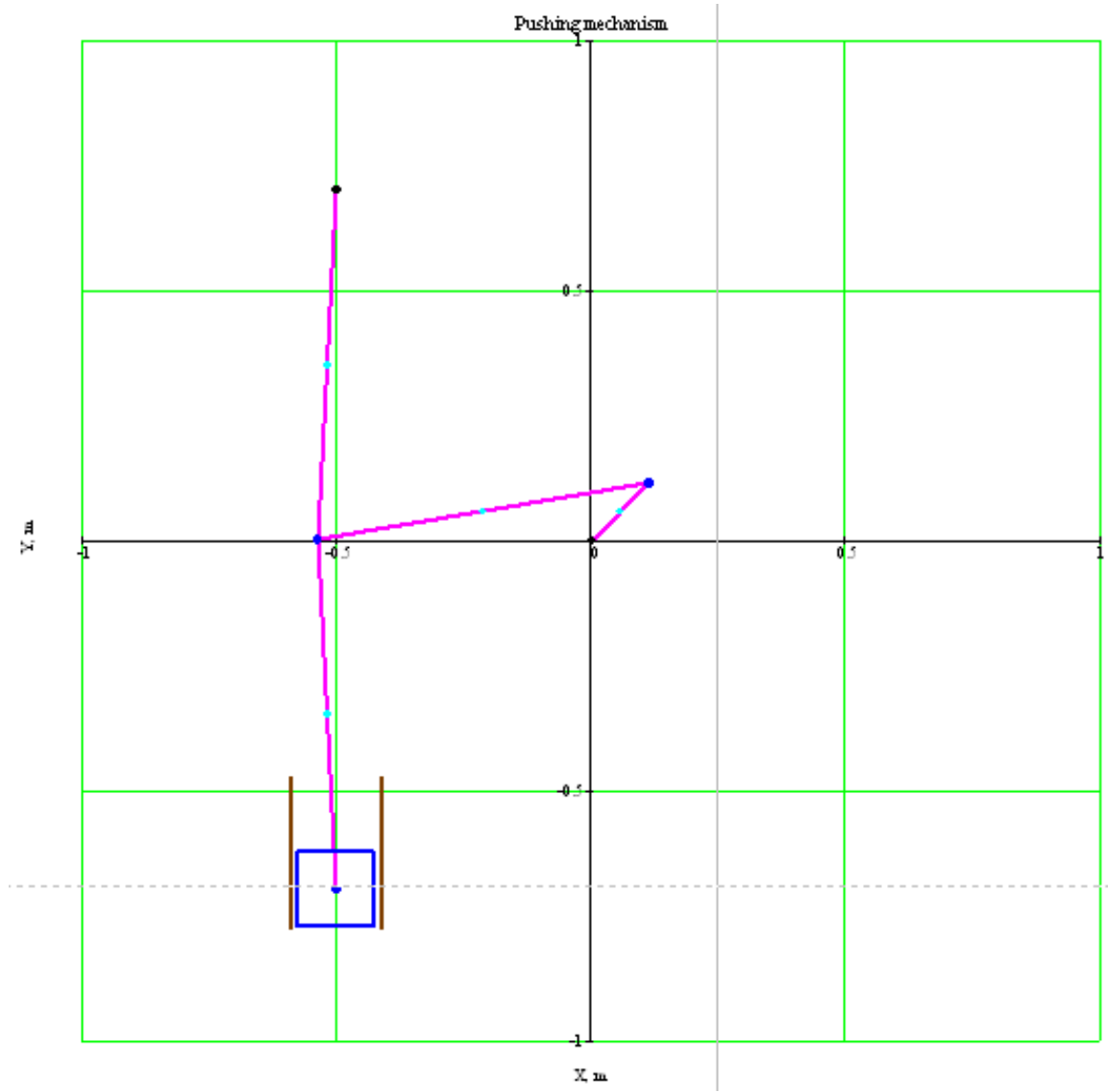

Рис.3. Мить анімації важільного механізму преса при $\alpha_{1}=45^{\circ}$.

У роботі застосовано методи теорії механізмів і машин, обчислювальної математики та моделювання в системі Mathcad. Отримані, в системі Mathcad, програма анімації та анімація [7] може застосовуватись на практичних, лекційних заняттях, при розрахунку, конструюванні та дослідженні кривошипно - повзунного механізму преса.

Слід сказати про те, що проведення комп’ютерного моделювання має ряд переваг: 
- не потрібно використовувати дороге лабораторне обладнання;

- скорочується час дослідження;

- вільно управляти процесом моделювання (переривати, відновлювати, змінювати умови або параметри).

Подальший напрям роботи передбачає комп’ютерну анімацію векторів швидкостей, прискорень та динамічних навантажень ланок досліджуваного механізму.

\section{Список використаних джерел}

1. Кіницький Я. Т. Теорія механізмів і машин в системі Mathcad: навчальний посібник/ Я. Т. Кіницький, В. О. Харжевський, М. В. Марченко. М. - Хмельницький: РВЦ ХНУ, 2014. - $324 \mathrm{c}$.

2. Бертяев В.Д. Теоретическая механика на базе Mathcad: практикум / В.Д. Бертяев. - СПб.: БХВ - Петербург, 2005. - 762 с.

3. Бойчук И.П. Комплексный подход к преподаванию теоретической механики с использованием информационно-коммуникационных технологий / И. П. Бойчук, О. И. Морозова, Т. В. Бойчук // Інформаційні технології і засоби навчання. - 2014. - Т. 41, Вип. 3. C. 128-141.

4. Рыков В.Т. Интерактивные элементы обучения в электронном задачнике по теоретической механике [Електронний ресурс] / В.Т. Рыков, Е.Н. Буряк, Е.В. Рыкова // Успехи современного естествознания. - 2005. - № 6. - С. 52-53. Режим доступу: http://www.natural-sciences.ru/ru/article/view?id=8636 (Дата обращения: 05.12.2017).

5. Панина Т.С. Современные способы активизации обучения / Т.С. Панина, Л. Н. Вавилова; под. ред. Т.С. Паниной. - 4-е изд., стереотип. - М.: Академия, 2008. - 176 с.

6. Герасимов В.В. Засоби комп'ютерного моделювання в навчанні та практичній підготовці інженерів у галузі легкої промисловості / В.В. Герасимов, М.І. Ігнатишин // Освіта i формування конкурентоспроможності фахівців в умовах євроінтеграції: збірник тез доповідей II Міжнародної науково-практичної конференції (25-26 жовтня 2018 р., Мукачево) / ред.кол.: Т.Д. Щербан (гол.ред.) та ін. - Мукачево: Вид-во МДУ, 2018. - С.489.

7. Важільний механізм преса. Варіант 2 [Електронний ресурс]. - Режим доступу: https://youtu.be/vIhmj3fDIzU

\section{References}

1. Ya. T. Kinyts'kyy, V. O. Kharzhevs'kyy and M. V. Marchenko, Teoriya mekhanizmiv $i$ mashyn $v$ systemi Mathcad [Theory of mechanisms and machines in the Mathcad system]. Khmelnytsky: RVC KhNU, 2014.

2. V.D. Bertyaev, Teoretycheskaya mekhanyka na baze Mathcad [Theoretical mechanics based on Mathcad]. St. Petersburg: BHV - Petersburg, 2005.

3. Y. P. Boychuk, O. Y. Morozova, T. V. Boychuk, "Kompleksnыy podkhod k prepodavanyyu teoretycheskoy mekhanyky $\mathrm{s}$ yspol'zovanyem ynformatsyonnokommunykatsyonnыkh tekhnolohyy [An integrated approach to the teaching of theoretical mechanics with the use of information and communication technologies] ", Information technology and learning tools, vol.41, no. 3, pp. 128-141, 2014.

4. V.T. Rykov, E.N. Buryak, E.V. Rykova "Ynteraktyvnye эlementy obuchenyya v эlektronnom zadachnyke po teoretycheskoy mekhanyke [Interactive elements of education in the electronic book of problems on theoretical mechanics] ", Advances in modern science, №6, pp. 5253, 2005. [Electronic resource]. Available at: http://www.natural-sciences.ru/ ru/article/ view?id=8636. Application date :05.12.2017.

5. T.S. Panyna, L. N. Vavylova, Sovremennye sposoby aktyvyzatsyy obuchenyya [Modern ways to enhance learning]. Moscow: Academy, 2008.

6. V.V. Herasymov, M.I. Ihnatyshyn, “Zasoby komp'yuternoho modelyuvannya v navchanni ta praktychniy pidhotovtsi inzheneriv $\mathrm{u}$ haluzi lehkoyi promyslovosti [Computer 
simulation tools in the studying and practical training of engineers in light industry] ", in II International Scientific and Practical Conference. Education and competitiveness formation of specialists in the conditions of European integration, Mukachevo, 2018, P.489.

7. Vazhil'nyy mekhanizm presa. Variant 2 [The lever mechanism of the press. Option 2] [Online]. Available: https://youtu.be/vIhmj3fDIzU

УДК 622. 3

DOI: 10.31339/2617-0833-2018-2(25)-38-44

ОБОСНОВАНИЕ ОСНОВНЫХ ПАРАМЕТРОВ СИСТЕМЫ АВТОМАТИЧЕСКОГО УПРАВЛЕНИЯ УГЛЕДОБЫВАЮЩИМИ КОМБАЙНАМИ В ПРОФИЛЕ ПЛАСТА Каганюк О.К., Полищук М.М., Гринюк С.В.

\section{SUBSTANTIATION OF THE MAIN PARAMETERS OF AUTOMATIC CONTROL SYSTEMS FOR COAL-MINING COMBINES IN THE FORMATION PROFILE}

Kaganjuk Alexey, Polischuk Nicholas, Hrynjuk Sergey

При разработке угольных месторождений угледобывающие комбайны работают в сложных горно-геологических условиях добычи угля. В столь трудных условиях работы машинисту комбайна приходится контролировать положение режущих органов (РО) работающих как по почве, так и по кровле угольного пласта. Данная ситуация приводит к значительным потерям угля, ухудшает качество добытого полезного ископаемого $u$ повышает травматизм технического персонала, обслуживающего угледобывающий комбайн. В данной статье даны обоснования по формированию основных требований и функиий качества для создания систем автоматического управления (САУ ПП) угледобывающими комбайнами в профиле пласта.

Ключевые слова: система автоматического управления, обоснование основных параметров, безлюднаявыемкаугля

When mining coal deposits, coal miners work in difficult mining and geological conditions of coal mining. In such difficult working conditions, the machine operator of the combine has to control the position of the cutting organs (RO) working both on the soil and on the roof of the coal seam. This situation leads to significant losses of coal, worsens the quality of mined minerals and raises the traumatism of technical personnel serving the coal mining combine. In this article, we give justifications on the formation of the basic requirements and quality functions for the creation of automatic control systems (SAU PP) by coal-mining combines in the formation profile.

Keywords:automaticcontrolsystem, substantiationofthemain parameters, desertedcoal.

Одной из важнейших подсистем управления угледобывающими комбайнами является подсистема автоматического управления в профилепласта (САУ ПП). До настоящего времени поставленная задача по решению данного вопроса не выполнена вопреки малоизученному процессу построения такого рода систем, где бы были изучены и сформулированы основные положения по формированию реальных требований к САУ ПП. Это является актуальным в решении безопасности ведения горных работ в условиях горногеологических нарушений, поскольку машинисту комбайна постоянно приходится находиться рядом с угледобывающим комбайном, работающим в зоне повышенной опасности.

Проблема в решении поставленной задачи заключается не только в создании систем автоматического управления комбайнами в профиле пласта, но и в создании надежных технических средств контроля и считывания текущей информации о текущем положении как самого комбайна, так и положение режущих органов (РО) относительно границы раздела 